\title{
Dosimetric quantities of neuroendocrine tumors over treatment cycles with ${ }^{177} \mathrm{Lu}$-DOTA-TATE
}

Daniel Roth ${ }^{1 *}$, Johan Gustafsson ${ }^{1 *}$, Carl Fredrik Warfvinge ${ }^{2}$, Anna Sundlöv ${ }^{1,2}$, Anna Åkesson ${ }^{3}$, Jan Tennvall ${ }^{2}$, Katarina Sjögreen Gleisner ${ }^{1}$

* Shared first authorship

1. Department of Medical Radiation Physics, Clinical Sciences Lund, Lund University, Lund, Sweden

2. Division of Oncology and Pathology, Department of Clinical Sciences Lund, Lund University, Lund, Sweden

3. Clinical Studies Sweden - Forum South, Skåne University Hospital, Lund, Sweden

\section{Corresponding Author:}

Katarina Sjögreen Gleisner

Department of Medical Radiation Physics

Barngatan 4

SE-221 85 Lund, SWEDEN

Phone: +46 (0)46-173133

E-mail: katarina.sjogreen_gleisner@med.lu.se

Word count: 5203

Short running title: Dosimetry across ${ }^{177} \mathrm{Lu}$-DOTA-TATE cycles 
ABSTRACT

Tumor dosimetry was performed for ${ }^{177} \mathrm{Lu}$-DOTA-TATE with the aims of better understanding $i$ ) the range and variation of the tumor absorbed doses (ADs), ii) how different dosimetric quantities evolve over the treatment cycles, and iii) whether this evolution differs depending on the tumor grade. Such information is important for radiobiological interpretation and may inform the design of alternative administration schemes. Methods: Data come from 41 patients with neuroendocrine tumors (NETs) of grade 1 ( $n=23$ ) or $2(n=18)$, that had received between 2 and 9 treatment cycles. Dosimetry was performed for 182 individual lesions, giving in total 880 individual AD assessments across all cycles. Hybrid planar-SPECT/CT imaging was used, including quantitative SPECT reconstruction, voxel-based absorbed-dose-rate calculation, semiautomatic image segmentation, and partial-volume correction. Linear mixed-effect models were used to analyze changes over cycles in tumor ADs, absorbed-dose rates and activity concentrations at day-1, effective half-times, and tumor volumes. Tumors smaller than $8 \mathrm{ml}$ were excluded from analyses. Results: Tumor ADs ranged between 2 and 77 Gy per cycle. On average the AD decreased over the cycles, with significantly different rates $(p<0.05)$ for grade 1 and 2 NETs of $6 \%$ and $14 \%$ per cycle, respectively. The absorbed-dose rates and activity concentrations at day-1 decreased by similar amounts. The effective halftimes were less variable but shorter for grade 2 than grade $1(p<0.001)$. For grade 2 NETS the tumor volumes decreased, with a similar tendency in grade 1. Conclusions: The tumor AD, absorbed-dose rate and activity uptake decrease, in parallel with tumor volumes, between ${ }^{177}$ Lu-DOTA-TATE treatment cycles, particularly for grade 2 NETs. The effective half-times vary less but are lower for grade 2 than grade 1 NETs. These results may indicate the development of radiation-induced fibrosis and could have implications for the design of future treatment and dosimetry protocols.

Key Words: ${ }^{177}$ Lu-DOTA-TATE; neuroendocrine tumors; absorbed dose; fractionation 


\section{INTRODUCTION}

${ }^{177} \mathrm{Lu}$-DOTA-TATE is well-established for the treatment of neuroendocrine tumors (NETs). A protocol of 4 cycles of $7.4 \mathrm{GBq}$ administered at an interval of approximately 2 months has been proven safe and effective (1-3). Since the therapy is based on ionizing radiation, the likelihood of tumor response is expected to be related to the absorbed dose (AD) (4-11). Current data indicate that the tumor ADs vary considerably between patients, from a few grays to nearly 200 Gy $(8,9,12-15)$. Most studies on tumor dosimetry have focused on a single lesion in each patient, and there is currently limited data on the variation in AD between metastases within patients, and variation across treatment cycles. Such information is important to advance the understanding of how the AD delivery is currently fractionated, and to inform the design of alternatives to the standard administration scheme where the activity per cycle, number of cycles, or time between cycles are modified $(4,7-9,11,16)$.

The AD is essentially calculated from a combination of an initial absorbed-dose rate and an effective half-time, in turn derived from estimates of the activity concentration over time. Understanding changes in these input quantities is essential for a deeper understanding of any systematic changes in the AD. Such information is also important from a practical perspective. A varying initial absorbed-dose rate but a stable half-time implies that the latter needs to be determined at cycle one only, thereby simplifying the dosimetry protocol.

Tumor biology adds another level of complexity. Gastroenteropancreatic NETs are divided into grades G1, G2, and G3 based on Ki67 staining representing the proliferation rate. G1 tumors (Ki67<3\%) are indolent, while the G3 tumors (Ki67> 20\%) are more aggressive. G2 tumors have a proliferation rate of 3 to $20 \%$ with a moderate aggressiveness. Additionally, there is an inverse relationship between somatostatin-receptor expression and grade (17).

The aim of this work is to improve the understanding of how the tumor AD is delivered over the treatment cycles, including effect of tumor grade. Further aims are to elucidate which underlying quantity is mainly responsible for any changes in the tumor ADs, and whether dosimetry can be simplified. 


\section{MATERIAL AND METHODS}

\section{Patient Data}

Data were obtained from the Iluminet trial (NCT01456078), for which a detailed description has been published elsewhere (18). The Iluminet trial was a phase II, non-randomized clinical trial that included 103 patients at two sites in Sweden 2011 to 2018. The trial included patients with somatostatin-receptorexpressing NETs of gastroenteropancreatic or bronchopulmonary origin, with a Ki67-index of up to $20 \%$ (G1 and G2) based on the most recent biopsy prior to inclusion in the study. They were given repeated cycles of ${ }^{177}$ Lu-DOTA-TATE $(7.4 \mathrm{GBq})$ at intervals of $(10 \pm 2)$ weeks. Treatment was continued up to a renal biologically effective dose $(\mathrm{BED})$ of $(27 \pm 2)$ Gy unless tumor progression or treatment-limiting toxicity occurred earlier. Patients with a good renal and hematological tolerance, and no signs of tumor progression, were offered to continue up to a renal BED of $(40 \pm 2) \mathrm{Gy}$. Final analysis of the trial results is currently underway. This study is based on a subset of the Iluminet patients, acquired at one of the study sites, and includes 41 patients who received two to nine cycles.

\section{Image Data}

Two SPECT/CT systems were used, a GE Discovery VH and a GE Discovery 670 (GE HealthCare). Images were acquired using medium-energy collimators and energy-windows centered at $208 \mathrm{keV}$, with widths 20\% (GE Discovery VH) or 15\% (GE Discovery 670).

Whole-body images were acquired at nominal times 1 h, 24 h (day-1), 96 h (day-4), and $168 \mathrm{~h}$ (day7) post administration. The gamma-camera images were co-registered to an X-ray scout to enable pixelwise attenuation and scatter correction (19).

SPECT/CT images were acquired at day-1, with 60 projections each of $45 \mathrm{~s}, 128 \times 128$ matrix, and pixel size $4.42 \times 4.42 \mathrm{~mm}^{2}$ (Discovery 670 ) or $4.02 \times 4.02 \mathrm{~mm}^{2}$ (Discovery VH). The low-dose CT images were rescaled to mass-density using a two-segment linear function based on calibration 
measurements. For each set of projection data, three different settings were used for the OS-EM reconstruction (see overview of image data analysis in supplement 1). The reason for this three-fold reconstruction was that different steps in the dosimetry process required images with different characteristics $(20,21)$. The first reconstruction (AS-8) was used for segmentation and employed 8 iterations and 6 angles per subset with compensation for attenuation and scatter (22). The second reconstruction (ASR-8), used for visual inspection, also included distance-dependent resolution compensation. The third reconstruction (ASR-40) was used for image-based quantification with 40 iterations and 6 angles per subset with compensations for attenuation, scatter, and resolution. All SPECT images were calibrated to reflect the activity per voxel by application of a calibration factor from measurements in air (23). Absorbed-doserate maps were calculated from the ASR-40 SPECT images using a voxel-based Monte Carlo program based on the EGS4 code with PRESTA $(23,24)$.

Recovery coefficients (RCs) were determined by phantom measurements, of an elliptical waterfilled Jaszczak phantom with 12 spherical inserts with ${ }^{177}$ Lu-DOTA-TATE with volumes of 3.9 to $93 \mathrm{ml}$. Four spheres were from a commercial vendor, while eight spheres were 3D-printed by fused filament fabrication. Images were acquired for one to three spheres at a time using the same acquisition settings as for patients and reconstructed as ASR-40. The RC was calculated as the ratio of the sphere activity estimated from images and the activity from phantom preparation, and a function of the RC versus volume was fitted (21).

Image Analysis

Tumors were delineated in the SPECT images using a semi-automatic 3D segmentation method based on Fourier surfaces (21). Initialization was made by manual delineation of volumes-of-interest (VOIs) that roughly encompassed the tumor, using the ASR-8 SPECT image for guidance. A closed surface was adapted to the tumor boundary as represented by high image gradients in the AS- 8 SPECT images, which was earlier shown to give a good volume preservation (21). The VOI was applied in the ASR-40 images, 
and the mean activity concentration calculated. The mean absorbed-dose rate was determined by applying the same VOI in the absorbed-dose-rate maps. The RC for the VOI volume was applied to both the activity concentration and the absorbed-dose rate.

Planar images were analyzed by identifying the tumors using an in-house graphical user-interface. Segmentation was performed using a semi-automatic method (20), which, when applied in the timesequence of images yielded tumor-specific time-activity data (in relative units). Absorbed-dose rate as a function of time was determined by rescaling the planar-derived data to the SPECT-derived absorbed-dose rate at day-1. A curve was fitted, consisting of a mono-exponential function for the three last data points and a quadratic function for the early phase (20). The $\mathrm{AD}$ was obtained by analytical integration of the absorbed-dose rate.

The SPECT VOIs and planar ROIs were verified by the responsible oncologists using diagnostic images, e.g., contrast-enhanced CT or MRI. In a few cases the VOIs and ROIs were adjusted or redelineated, using the Otsu method (25) or a manually selected threshold. Verification also aimed at ensuring that the planar-derived data fulfilled the validity criteria described in (20).

To make image-derived quantities consistent for statistical analysis the ADs, absorbed-dose rates, and activity concentrations were re-calculated to a nominal administered activity of 7.4 GBq. The absorbeddose rates and activity concentrations were also adjusted to a reference time-point, set to the average timepoint of all SPECT/CT images acquired day-1 $(21.7 \pm 1.5 \mathrm{~h})$. This adjustment was made based on the effective half-time for the individual tumor, or if unavailable, the mean half-time for other tumors within the same patient, or else across all patients. Different exclusion criteria were set for the analyses of different quantities. A volume cut-off of $8 \mathrm{ml}$ was applied to avoid the bias observed for small volumes (21). For the $\mathrm{AD}$ and effective half-time, tumors that suffered from substantial superposition of activity in the planar images were excluded (20). An additional criterion was set for the effective half-time, to exclude cycles where all four planar images, for practical reasons, were not acquired. 
Different alternatives of simplifying the dosimetry process were evaluated in terms of how well the cumulative $\mathrm{AD}$ across all cycles could be estimated. As reference, tumors that met the selection criteria for all cycles were identified and the cumulative ADs determined. These were compared to the cumulative ADs when assuming a constant $\mathrm{AD}$ estimated from the first cycle (A), a constant half-time estimated from the first cycle (B), or as the grade-specific mean (C). Both (B) and (C) were combined with a cycle-specific estimate of the absorbed-dose rate, assuming mono-exponential washout. The possibility to use the fitted LMM (below) for interpolation of missing cycle data was also explored (D), and as a consistency check, the cumulative ADs obtained from Eq. 1 (E). Evaluation was made by means of Bland-Altman plots (supplement 3).

\section{Statistical Analysis}

Analysis of changes over the treatment cycles was made using a linear mixed-effect model (LMM) in R version 4.0.2 (26,27). Dependent variables were the $\mathrm{AD}$, the absorbed-dose rate at day-1, the activity concentration at day-1, the effective half-time, and the tumor volume. The model was

$$
Q(n, g)_{i, j}=\exp \left[q_{1}+q_{2} \cdot g+n \cdot\left(k_{1}+k_{2} \cdot g\right)+\Delta q_{i}+\Delta k_{i} \cdot n+\Delta q_{i, j}+\Delta k_{i, j} \cdot n\right]
$$

where $n$ was the cycle number, $g$ the grade status for the patient (G1: $g=0, \mathrm{G} 2: g=1)$, and $Q(n, g)_{i, j}$ the dependent variable for tumor $j$ in patient $i$. Parameters $q_{1}$ and $q_{2}$ described the global intercepts, and $k_{1}$ and $k_{2}$ the mean rates-of-change with respect to the cycle number, for G1 and G2. These parameters were treated as fixed effects. Parameters $\Delta q_{i}, \Delta k_{i}, \Delta q_{i, j}$, and $\Delta k_{i, j}$ constituted the random effects, where $\Delta q_{i}$ and $\Delta k_{i}$ described the patient-specific differences from the global intercepts and rates-of-change, while $\Delta q_{i, j}$, and $\Delta k_{i, j}$ described the tumor-specific differences from the sum of the fixed effects and the patient- 
specific random effects. The 95\% confidence intervals (CIs) for the rates-of-change and the difference between $\mathrm{G} 1$ and $\mathrm{G} 2$ were also derived (28).

The mean value across all cycles was calculated using a second LMM. This was constructed by omitting the cycle-dependent terms in Eq. 1, according to

$$
\bar{Q}(g)=q_{1}+q_{2} \cdot g+\Delta q_{i}+\Delta q_{i, j}
$$

The global mean for G1 and G2 were thus $q_{1}$ and $\left(q_{1}+q_{2}\right)$, respectively. Confidence intervals were constructed using the Confint function in R.

\section{RESULTS}

Of the G1 patients $(n=23)$ the primary-tumor origin was pancreas $(n=1)$, small intestine/right colon $(n=20)$, and lung $(n=2)$. For G2 patients $(n=18)$ it was pancreas $(n=7)$, small intestine/right colon $(n=9)$, lung $(n=1)$, and unknown $(n=1)$.

Altogether, dosimetry was performed for 182 tumors in 41 patients given between 2 and 9 treatment cycles, resulting in $880 \mathrm{AD}$ assessments. Of these, the criterion for activity concentration and absorbeddose rate analysis was fulfilled for 500 data points, representing 138 tumors in 40 patients. From these, 404 fulfilled the criteria for AD estimation, representing 109 tumors in 39 patients. The criteria for the effective half-time were fulfilled in 301 cases, representing 104 tumors in 39 patients. As a result of the inclusion criteria, each patient and tumor were not represented at every cycle.

Figure 1 shows examples of SPECT VOIs from the semi-automatic segmentation that in the majority of cases yielded results consistent with image data (21). The RC curve is shown in Figure 2. The in-house manufactured spheres provided a relevant volume range with respect to partial-volume correction.

Figure 3 summarizes the AD per cycle, its variability, and how the variability is decomposed over cycles for individual tumors, between tumors and between patients. The tumor AD ranged between 2 and 
77 Gy and there was substantial variation both between cycles and between tumors. The median AD for G1 patients was $21 \mathrm{~Gy}$ (quartiles 13 and 41, range 3.5 to $66 \mathrm{~Gy}$ ). For G2 patients the median was 13 Gy (quartiles 7.4 and 24, range 4.7 to 32 Gy). The overall difference between G1 and G2 was mainly caused by a more pronounced $\mathrm{AD}$ decrease over the cycles for G2 (Figure 4). For G1, the medians for cycles 1 to 5 were 33, 33, 30, 26, and 28 Gy, respectively, and 24 Gy for 6 cycles or more. For G2, medians for cycles 1 to 5 were $27,23,15,12,8 \mathrm{~Gy}$, respectively, and 6 Gy for 6 cycles or more.

Figure 5 shows examples of the AD evolution for individual tumors over treatment cycles and how the LMM (Eq. 1) decomposes the intercepts and rates-of-change with respect to the individual patient and tumor. The fixed-effect intercepts over all patients and tumors for G1 $\left(\exp \left(q_{1}\right)\right)$ and G2 $\left(\exp \left(q_{1}+q_{2}\right)\right)$ were 24.6 and $19.4 \mathrm{~Gy}$, respectively, and were not significantly different $(p=0.3)$. The changes between consecutive cycles are summarized in Table 1 . On average, the AD rate-of-change for G2 was -0.15 , corresponding to a $14 \%$ decrease between consecutive cycles. For G1 the decrease was less pronounced, with a mean rate-of-change of -0.06 , or $6 \%$ decrease between cycles, and the upper limit of the confidence interval was near zero. The AD changes of G1 and G2 were significantly different.

The activity concentration exhibited a similar decrease as the AD (Figure 6) as did the absorbeddose rate (Table 1). The median absorbed-dose rates for G1 were 187, 175, 163, 136, 133, and $127 \mathrm{mGy} / \mathrm{h}$ for cycles 1 to 5 , and 6 cycles or more, respectively, while for G2 they were $184,167,125,87,58$, and 50 $\mathrm{mGy} / \mathrm{h}$. The decreased AD over the cycles was thus associated with a decreased absorbed-dose rate, in turn governed by a decreasing activity concentration. The tumor volumes did not change for G1, while for G2 a per-cycle decrease of $6 \%$ was observed (Table 1). The fixed-effect intercepts were $20 \mathrm{ml}$ and $16 \mathrm{ml}$ for G1 and G2 patients, respectively, and were not significantly different $(p=0.4)$. The distributions of all tumor volumes at cycle 1 are shown in supplement 2. For the effective half-time, a cycle-dependent change was not observed (Table 1, Figure 6). The average over all patients, all cycles and all tumors (Eq. 2) were 103 h for G1 (CI: 96 to 109 h), and 81 h (CI: 73 to 90 h) for G2. The difference between G1 and G2 was significant $(p<0.001)$. 
The cumulative ADs obtained using complete dosimetry and the simplification alternatives are shown in Table 2 and supplement 3. The median cumulative AD was $137 \mathrm{~Gy}$ (range 33 to $403 \mathrm{~Gy}$ ) for G1 and 80 Gy (range 11 to 211 Gy) for G2. The assumption of a constant effective half-time across cycles yielded negligible systematic deviation both when estimated from the first cycle and as the grade-specific cohort means, while the limits-of-agreement were wider when using the population means. Assuming a constant $\mathrm{AD}$ across cycles yielded the largest deviations among the alternatives. The modest deviations obtained using Eq.1 confirmed the fit of the LMM to data.

\section{DISCUSSION}

We have found that when ${ }^{177}$ Lu-DOTA-TATE is given with a fixed activity in repeated cycles the tumor AD decreases over the course of treatment, especially for G2 NETs. This decrease is caused by a decreasing activity uptake in the tumors. We have also found that there is a large variability in tumor ADs, both between cycles for individual tumors, within patients, and between patients. This is an important observation for dosimetry-based treatment planning with the objective of reaching a minimum cumulative $\mathrm{AD}$ to tumors since it may be difficult to decide which tumor AD should guide treatment.

The observed decline in activity uptake and volume for G2 may be consistent with the development of radiation-induced stroma and fibrosis observed in pancreatic NET (29). For G1 the declines are less pronounced, which could possibly be related to the rate of cell necrosis (2). A low Ki67 index means that a higher proportion of cells are in the G0 phase of the cell cycle, and the progression to cell death may thus be slower for G1 than G2 NETs. This is also consistent with the published relationships between tumor shrinkage and cumulative $\mathrm{AD}$ at time of best response, which required longer follow-up time for smallintestinal NETs than for pancreatic NETs that generally have higher Ki67 $(8,9,11)$.

The effective half-times are not observed to change over the treatment cycles and are in rather narrow ranges, with significantly shorter half-times for G2 than G1. The lower ability to retain ${ }^{177} \mathrm{Lu}-$ DOTA-TATE for G2 NETs could be associated with tumor-cell necrosis, recruitment of immune cells and washout via the lymphatic system $(29,30)$. The limited variation in the effective half-times opens for 
simplifying the dosimetry method, by estimating the tumor-specific effective half-time for the first cycle and then assume an equal half-time for remaining cycles. The amplitude of the curve will still need to be estimated for each cycle but requires only one SPECT/CT.

A limitation in this study is that image segmentation and estimation of the tumor volume are based on SPECT, thereby making the VOI definition dependent on the activity distribution. The low-dose CT acquired as part of SPECT/CT does not provide sufficient quality for tumor identification, and the alternative would be to use contrast-enhanced CT and co-registration of the SPECT image. However, coregistration is known to introduce undesired interpolation effects and in view of the comparably small and differently located tumors this approach was not considered feasible. The volume cut-off of $8 \mathrm{ml}$ was applied to avoid negative bias in the activity concentration for smaller volumes (21). Another limitation is the use of planar imaging for assessing the effective half-time. In previous studies we have found good agreement with SPECT-derived data for tumors without substantial activity overlap in the planar images (20).

Linear mixed-effects models are suitable for analysis of data that have complex covariance structures, including longitudinal and hierarchical data. For this study, the rates-of-change are based on repeated measurements with variance components both between patients and tumors in the same patient. Technically, a linear function is fitted to the logarithm of the data (Eq. 1), thereby assuming an exponential relationship between the dependent variable and cycle number. We deemed a multiplicative change (e.g., $10 \%$ per cycle) to be more appropriate than an additive change (e.g., 3 Gy per cycle).

For future treatment optimization, the ADs to healthy tissues also need consideration. Based on previously reported renal dosimetry data $(18,31)$ we calculated tumor-to-kidney AD ratios as a function of cycle (Figure 7), giving values between 0.33 and 17. For consistency, the rate-of-change of the kidney AD was also analyzed by omitting the tumor-specific terms in Eq.1, giving CIs that covered zero for both G1 and G2. The decreasing tumor-to-kidney ratio, mainly observed for G2, is thus governed by the decreasing tumor AD. These results raise the question whether it would be more beneficial to administer fewer cycles 
with a higher activity for $\mathrm{G} 2$. However, the specifics of such a protocol would require detailed consideration of the tumor-to-kidney $\mathrm{AD}$ ratios, as well as the risk of hematologic and pituitary toxicity $(4,7,16,32)$.

\section{CONCLUSIONS}

The tumor AD decreases between treatment cycles in ${ }^{177} \mathrm{Lu}$-DOTA-TATE treatment. The trend is more pronounced for G2 NETs than for G1 NETs and is mainly governed by a decreased activity uptake. G2 NETs also exhibit a decreasing volume over the cycles. The effective half-times do not demonstrate a systematic trend but are on average lower for G2 than G1 NETs. These results have implications for the design of alternative administration schemes and dosimetry protocols in ${ }^{177}$ Lu-DOTA-TATE treatment.

\section{KEY POINTS}

Question: Does the tumor absorbed dose change over treatment cycles in therapy of neuroendocrine tumors with ${ }^{177}$ Lu-DOTA-TATE?

Pertinent findings: An exploratory analysis of 41 patients showed a decrease in tumor absorbed doses by $6 \%$ per cycle for G1 tumors and $14 \%$ per cycle for G2 tumors, both statistically significant. The per-cycle decrease was caused by a lower activity uptake in the tumors and a decreasing volume, while the effective half-times were less variable.

Implications for patient care: The results have implications for design of future administration protocols and for the implementation of tumor dosimetry ${ }^{177}$ Lu-DOTA-TATE therapy.

\section{DISCLOSURES}

AS has been a consultant for Novartis, Ipsen, and Spago Nanomedical. KSG has been a consultant for Fusion Pharmaceuticals Inc. 
Economic support was granted by the Swedish Cancer Society (contract 180747) and the Mrs. Berta Kamprad's Foundation (BKS-2020-13-293 and BKS-2020-8-270).

No other potential conflicts of interest relevant to this article exist.

\section{ACKNOWLEDGMENTS}

We wish to express our gratitude to the included patients, and co-workers Fanny Mörnsjö Centofanti, Paula Gluchowski, Cecilia Hindorf, Erik Larsson, Johanna Svensson and Peter Bernhardt. 


\section{REFERENCES}

1. Strosberg J, El-Haddad G, Wolin E, et al. Phase 3 trial of 177Lu-Dotatate for midgut neuroendocrine tumors. N Engl J Med. 2017;376:125-135.

2. Kwekkeboom DJ, de Herder WW, Kam BL, et al. Treatment with the radiolabeled somatostatin analog [177Lu-DOTA0,Tyr3] octreotate: toxicity, efficacy, and survival. J Clin Oncol. 2008;26:2124-2130.

3. Kim SJ, Pak K, Koo PJ, Kwak JJ, Chang S. The efficacy of (177)Lu-labelled peptide receptor radionuclide therapy in patients with neuroendocrine tumours: a meta-analysis. Eur J Nucl Med Mol Imaging. 2015;42:1964-1970.

4. Hagmarker L, Svensson J, Ryden T, et al. Bone marrow absorbed doses and correlations with hematological response during (177)Lu-DOTATATE treatments are influenced by image-based dosimetry method and presence of skeletal metastases. J Nucl Med. 2019;60:1406-1413.

5. Barone $\mathrm{R}$, Borson-Chazot $\mathrm{F}$, Valkema $\mathrm{R}$, et al. Patient-specific dosimetry in predicting renal toxicity with (90)Y-DOTATOC: relevance of kidney volume and dose rate in finding a dose-effect relationship. JNuclMed. 2005;46 Suppl 1:99S-106S.

6. Pauwels S, Barone R, Walrand S, et al. Practical dosimetry of peptide receptor radionuclide therapy with (90)Y-labeled somatostatin analogs. J Nucl Med. 2005;46 Suppl 1:92S-98S.

7. Sundlöv A, Sjögreen-Gleisner K, Tennvall J, et al. Pituitary function after high-dose 177LuDOTATATE therapy and long-term follow-up. Neuroendocrinology. 2020;Online First. 
8. Ilan E, Sandström M, Wassberg C, et al. Dose response of pancreatic neuroendocrine tumors treated with peptide receptor radionuclide therapy using 177Lu-DOTATATE. $J$ Nucl Med. 2015;56:177-182.

9. Jahn U, Ilan E, Sandström M, Garske-Roman U, Lubberink M, Sundin A. 177Lu-DOTATATE peptide receptor radionuclide therapy: dose response in small intestinal neuroendocrine tumors. Neuroendocrinology. 2020;110:662-670.

10. Chansanti O, Jahangiri Y, Matsui $Y$, et al. Tumor dose response in Yttrium-90 resin microsphere embolization for neuroendocrine liver metastases: a tumor-specific analysis with dose estimation using SPECT-CT. J Vasc Interv Radiol. 2017;28:1528-1535.

11. Jahn U, Ilan E, Sandström M, Lubberink M, Garske-Roman U, Sundin A. Peptide receptor radionuclide therapy (PRRT) with (177)Lu-DOTATATE; differences in tumor dosimetry, vascularity and lesion metrics in pancreatic and small intestinal neuroendocrine neoplasms. Cancers (Basel). 2021;13.

12. Cremonesi M, Ferrari ME, Bodei L, et al. Correlation of dose with toxicity and tumour response to (90)Y- and (177)Lu-PRRT provides the basis for optimization through individualized treatment planning. Eur J Nucl Med Mol Imaging. 2018;45:2426-2441.

13. Wehrmann C, Senftleben S, Zachert C, Muller D, Baum RP. Results of individual patient dosimetry in peptide receptor radionuclide therapy with 177Lu DOTA-TATE and 177Lu DOTA-NOC. Cancer Biother Radiopharm. 2007;22:406-416.

14. Kupitz D, Wetz C, Wissel H, et al. Software-assisted dosimetry in peptide receptor radionuclide therapy with 177Lutetium-DOTATATE for various imaging scenarios. PLoS One. 2017;12:e0187570. 
15. Rudisile S, Gosewisch A, Wenter V, et al. Salvage PRRT with (177)Lu-DOTA-octreotate in extensively pretreated patients with metastatic neuroendocrine tumor (NET): dosimetry, toxicity, efficacy, and survival. BMC Cancer. 2019;19:788.

16. Del Prete M, Buteau FA, Arsenault F, et al. Personalized (177)Lu-octreotate peptide receptor radionuclide therapy of neuroendocrine tumours: initial results from the P-PRRT trial. Eur J Nucl Med Mol Imaging. 2019;46:728-742.

17. Hofman MS, Lau WFE, Hicks RJ. Somatostatin receptor imaging with 68Ga DOTATATE PET/CT: clinical utility, normal patterns, pearls, and pitfalls in interpretation. RadioGraphics. 2015;35:500516.

18. Sundlöv A, Sjögreen-Gleisner K, Svensson J, et al. Individualised Lu-177-DOTATATE treatment of neuroendocrine tumours based on kidney dosimetry. Eur J Nucl Med Mol Imaging. 2017;44:1480-1489.

19. Sjögreen K, Ljungberg M, Strand SE. An activity quantification method based on registration of CT and whole-body scintillation camera images, with application to 131I. J Nucl Med. 2002;43:972-982.

20. Roth D, Gustafsson J, Sundlöv A, Sjögreen Gleisner K. A method for tumor dosimetry based on hybrid planar-SPECT/CT images and semiautomatic segmentation. Med Phys. 2018;45:5004-5018.

21. Gustafsson J, Sundlöv A, Sjögreen Gleisner K. SPECT image segmentation for estimation of tumour volume and activity concentration in (177)Lu-DOTATATE radionuclide therapy. EJNMMI Res. 2017;7:18. 
22. Frey EC, Tsui BMW. A new method for modeling the spatially-variant, object-dependent scatter response function in SPECT. 1996 IEEE Nuclear Science Symposium - Conference Record, Vols 1-3. 1997:1082-1086.

23. Ljungberg M, Frey E, Sjögreen K, Liu XW, Dewaraja Y, Strand SE. 3D absorbed dose calculations based on SPECT: Evaluation for 111-In/90-Y therapy using Monte Carlo simulations. Cancer Biother Radiopharm. 2003;18:99-107.

24. Bielajew AF, Rogers DWO. PRESTA - the Parameter Reduced Electron-Step Transport Algorithm for Electron Monte-Carlo Transport. Nucl Instrum Meth B. 1987;18:165-181.

25. Otsu N. A threshold selection method from gray-level histograms. IEEE Trans Syst Man Cybern. 1979;SMC-9:62-66.

26. R: A Language and Environment for Statistical Computing [computer program]. Version 4.0.2. Vienna, Austria: R Foundation for Statistical Computing; 2020.

27. Bates D, Mächler M, Bolker BM, Walker SC. Fitting linear mixed-effects models using lme4. Journal of Statistical Software. 2015;67:1-48.

28. emmeans: Estimated Marginal Means, aka Least-Squares Means [computer program]. Version 1.4.8. Vienna, Austria; 2020. 
29. Schiavo Lena M, Partelli S, Castelli P, et al. Histopathological and immunophenotypic changes of pancreatic neuroendocrine tumors after neoadjuvant peptide receptor radionuclide therapy (PRRT). Endocr Pathol. 2020;31:119-131.

30. Örbom A, Eriksson SE, Elgström E, et al. The intratumoral distribution of radiolabeled 177LuBR96 monoclonal antibodies changes in relation to tumor histology over time in a syngeneic rat colon carcinoma model. J Nucl Med. 2013;54:1404-1410.

31. Sundlöv A, Gustafsson J, Brolin G, et al. Feasibility of simplifying renal dosimetry in (177)Lu peptide receptor radionuclide therapy. EJNMMI Phys. 2018;5:12.

32. Bergsma H, van Lom K, Raaijmakers M, et al. Persistent Hematologic Dysfunction after Peptide Receptor Radionuclide Therapy with (177)Lu-DOTATATE: Incidence, Course, and Predicting Factors in Patients with Gastroenteropancreatic Neuroendocrine Tumors. J Nucl Med. 2018;59:452-458. 


\section{TABLES}

TABLE 1. Fixed-effect percent change from the previous cycle, calculated from the rates-of-change from the LMM, such that for G1 change $=\exp \left(k_{1}\right)-1$, and for G2 change $=\exp \left(k_{1}+k_{2}\right)-1$, with $k_{1}$ and $k_{2}$ as in Eq.1.

\begin{tabular}{lccccc}
\hline \multicolumn{7}{c}{ Change (\% from previous cycle) } \\
\hline & \multicolumn{3}{c}{ G1 } & \multicolumn{3}{c}{ G2 } & G1 vs G2 \\
\hline Dependent variable & Mean & CI & Mean & CI & $p$-value \\
\hline Activity concentration (MBq/ml) & -6.1 & $(-11,-0.89)$ & -14 & $(-20,-8.4)$ & 0.04 \\
Absorbed-dose rate (mGy/h) & -6.2 & $(-11,-0.93)$ & -14 & $(-20,-8.3)$ & 0.04 \\
Effective half-time (h) & -0.69 & $(-2.1,0.77)$ & -1.2 & $(-3.4,1.1)$ & 0.7 \\
Volume (ml) & -1.1 & $(-5.0,3.0)$ & -6.4 & $(-11,-1.4)$ & 0.1 \\
Absorbed dose (Gy) & -5.7 & $(-11,-0.12)$ & -14 & $(-20,-7.9)$ & 0.04 \\
\hline
\end{tabular}


TABLE 2. Relative difference in the cumulative AD for 65 individual tumors over 2 to 9 cycles, when introducing simplifications to the dosimetry protocol $(\mathrm{A}-\mathrm{C})$, using the LMM to interpolate missing cycle data (D), or the complete LMM (E). Relative difference is calculated as: (AD simplified protocol / AD complete dosimetry - 1). The limits of agreement (LOAs) are derived from Bland-Altman plots (supplement 3).

\begin{tabular}{lcc}
\hline Alternative & Mean & LOAs \\
& $(\%)$ & $(\%)$ \\
\hline A) Constant AD / cycle, from $1^{\text {st }}$ cycle & 15 & 55 \\
B) Constant effective half-time, from 1 ${ }^{\text {st }}$ cycle & 0.43 & 13 \\
C) Constant effective half-time, global means (G1: 103 h, G2: 81 h) & 0.01 & 31 \\
D) Interpolation, LMM intercept patient- and tumor-effective half-time (Eq.2) & 1.6 & 7.9 \\
E) Complete LMM for the effective half-time or AD (Eq. 1) & -1.0 & 3.3 \\
\hline
\end{tabular}




\section{FIGURES}
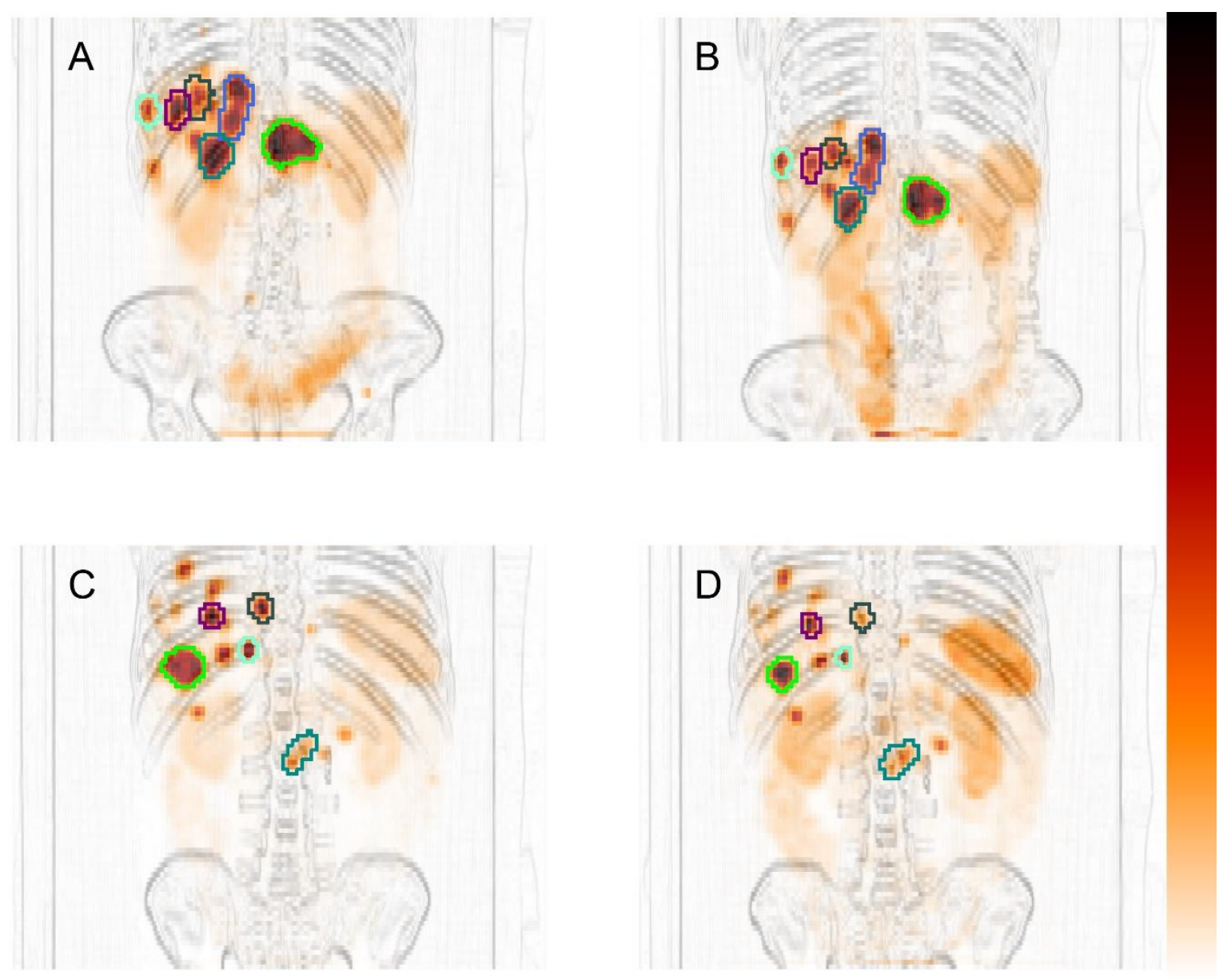

Figure 1. VOIs for cycle 1 (A, C) and cycle 4 (B, D) for one G1 NET (A, B) and one G2 NET patient (C, D). SPECT images are shown as maximum-intensity projections overlaid on high-pass-filtered maximumintensity projections of the CT. 


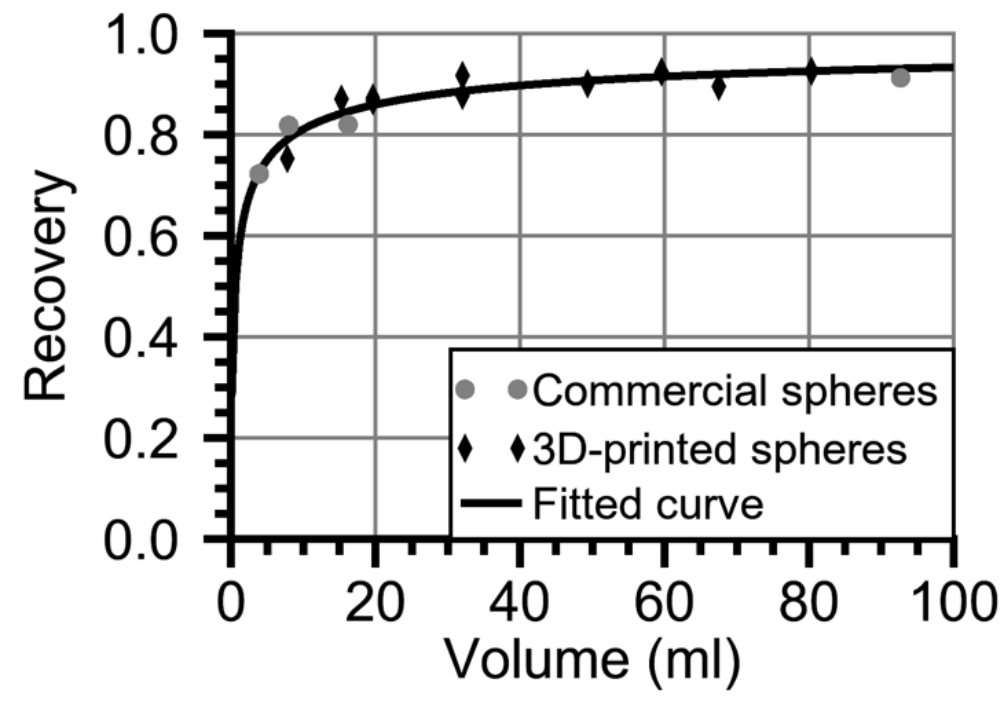

Figure 2. Measured recovery coefficients (RCs) and fitted curve. 


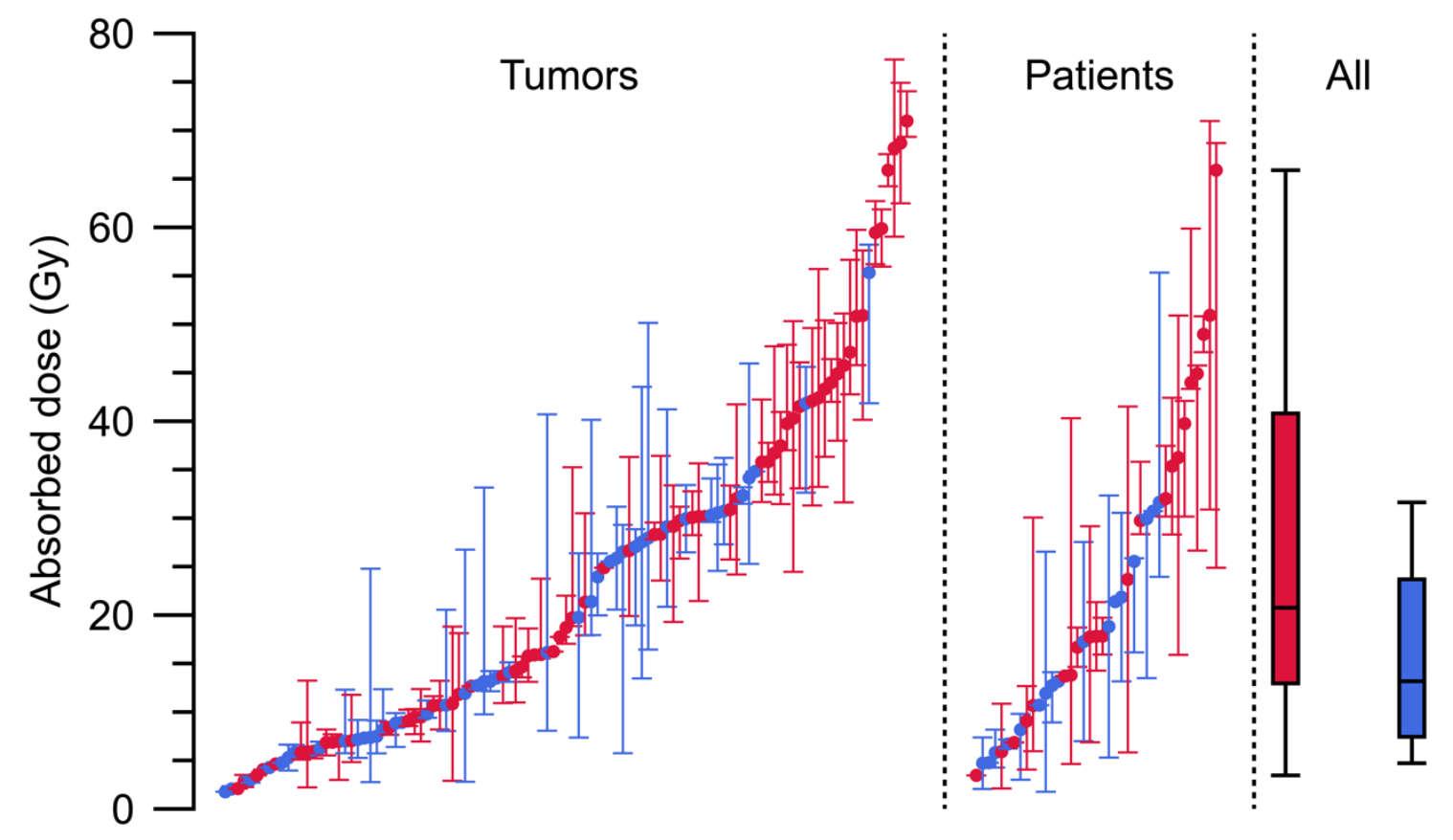

Figure 3. Dispersion of AD per 7,4 GBq to tumors over cycles (left), within patients (middle) and between patients (right), for G1 (red) and G2 NETs (blue). In tumor graph, dots represent the median AD over the cycles for each tumor, and whiskers the minimum and maximum AD. In patient graph, dots represent the median $\mathrm{AD}$ of the medians for tumors, and whiskers the minimum and maximum median $\mathrm{AD}$. All is a boxplot of the median, first and third quartiles, minimum and maximum of the median ADs for patients. 


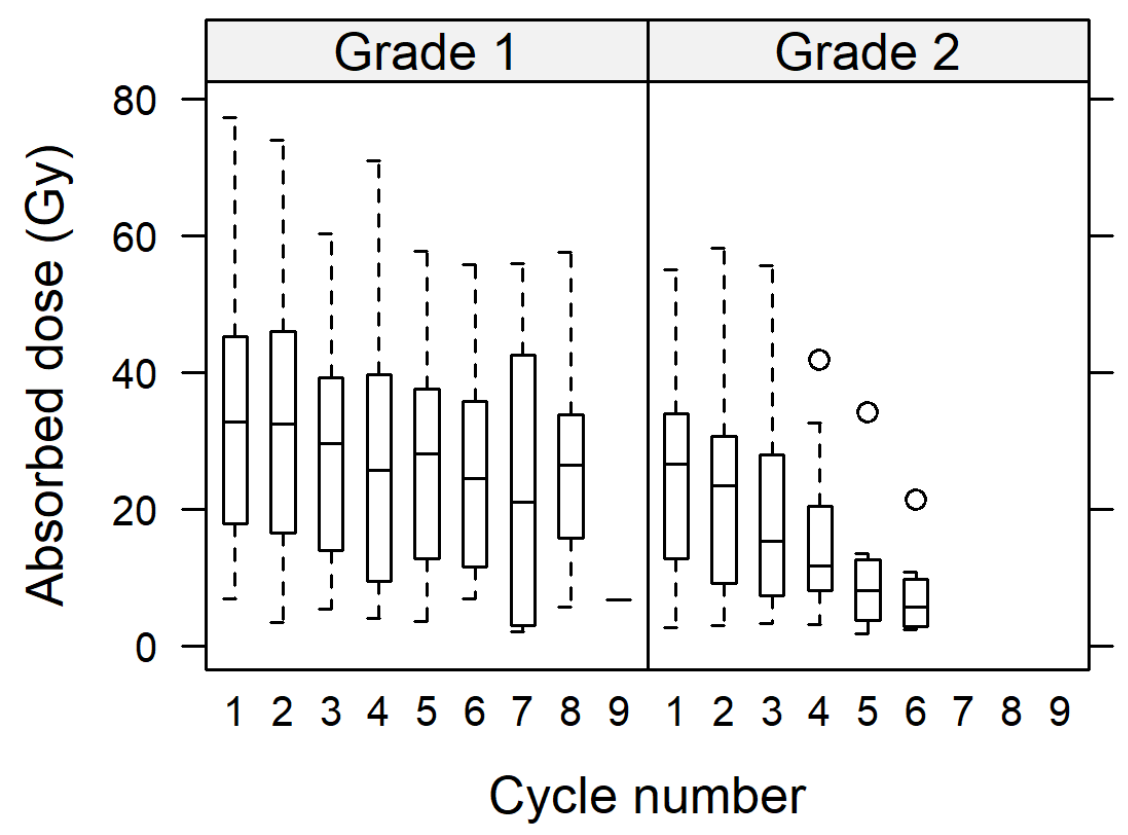

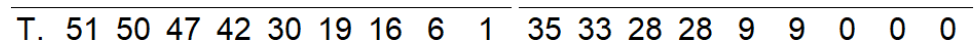

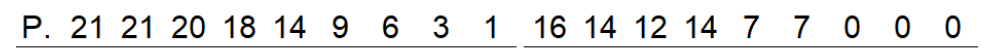

Figure 4. AD as function of cycle for G1 (left) and G2 NETs (right), across all patients and all tumors. Whiskers indicate 5th and 95th percentiles. Bottom: Number of tumors and patients underlying each box. 

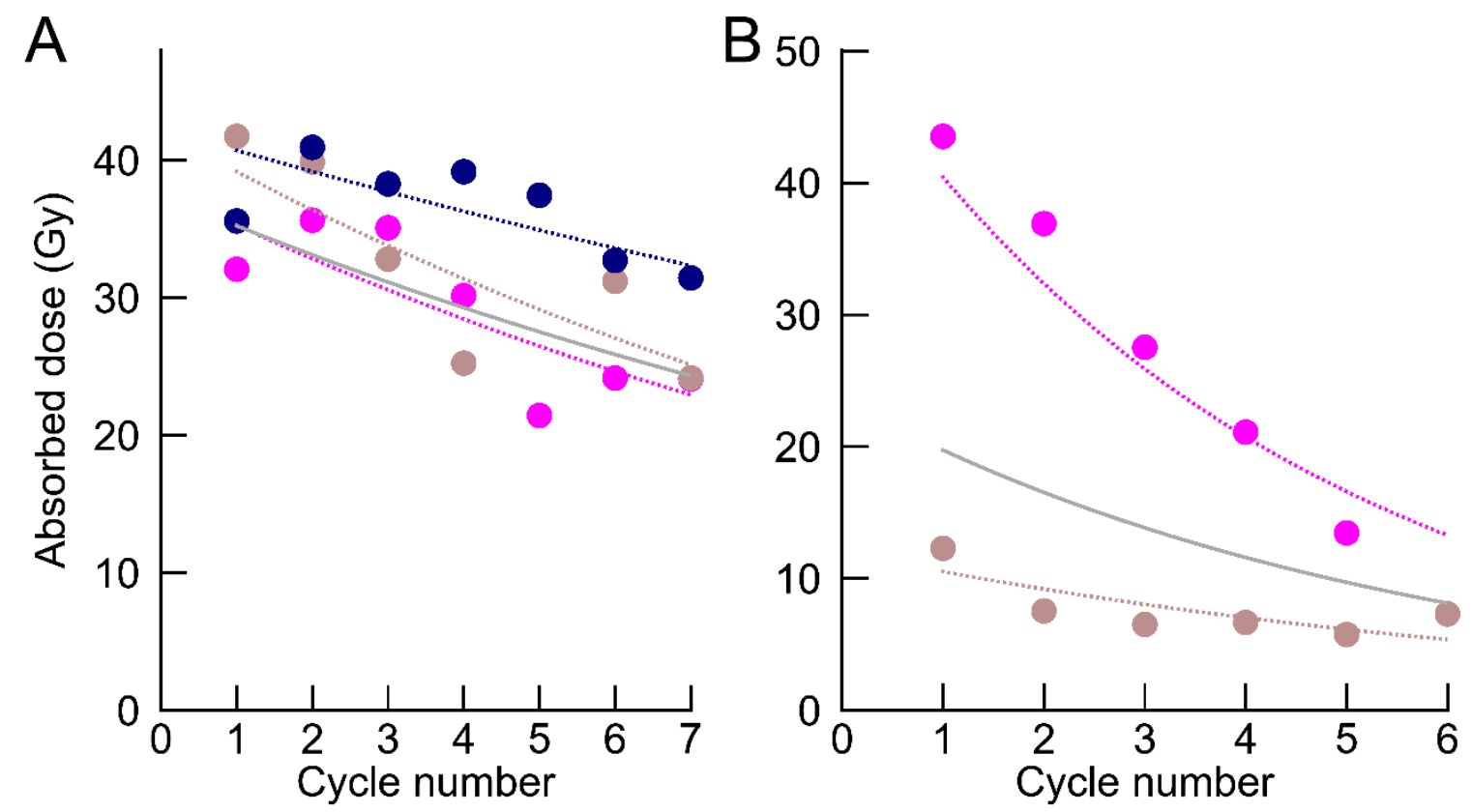

Figure 5. AD as function of cycle number (circles) for two patients, G1 (two tumors, A) and G2 NET (seven tumors, B). Gray solid lines show the fixed effects combined with the patient-specific random effects, each including an intercept and a rate constant (Eq. 1). Dashed lines are the tumor-specific curves, obtained as the sum of the fixed effects and the patient- and tumor-specific random effects. 

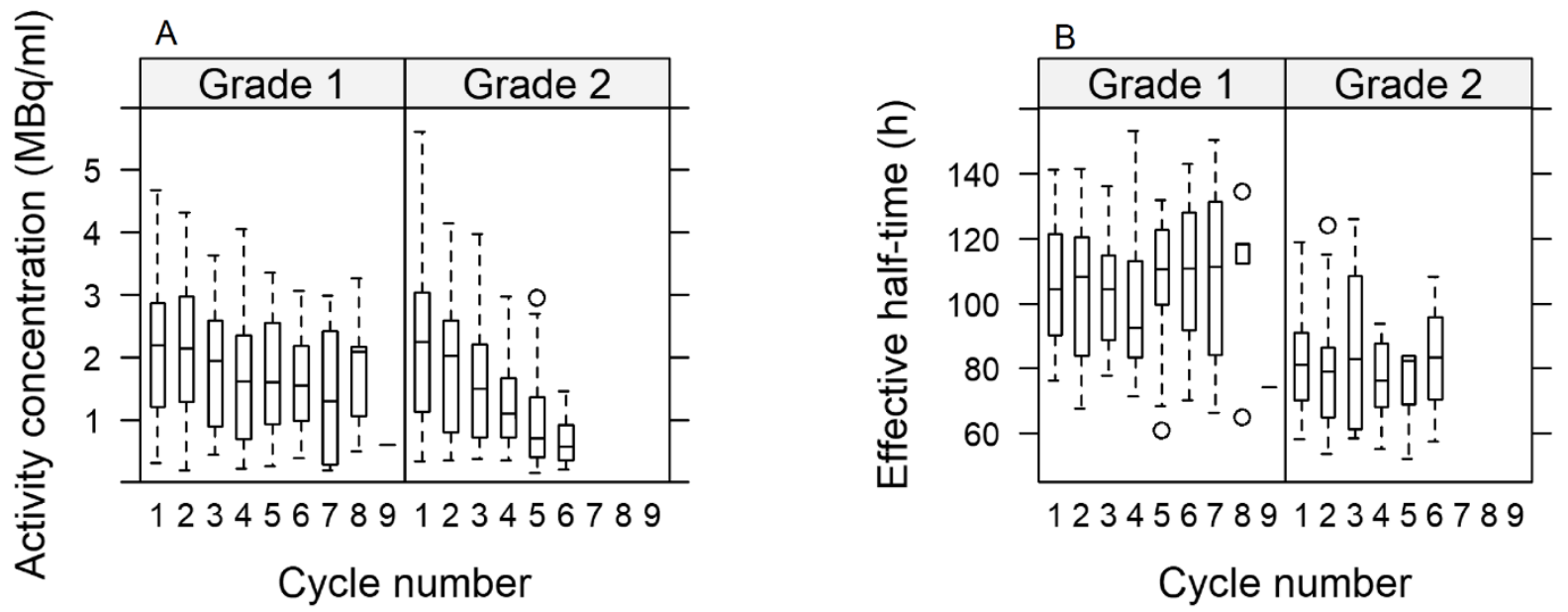

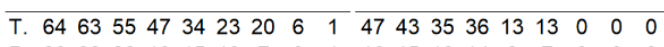

T. $\begin{array}{llllllllllllllllll}51 & 35 & 39 & 27 & 25 & 19 & 9 & 5 & 1 & 35 & 20 & 14 & 12 & 6 & 3 & 0 & 0 & 0\end{array}$

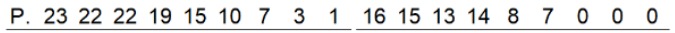

Figure 6. The activity concentration (A) and effective half-time (B) as functions of cycle number, for G1 and G2 NETs, across all patients and all tumors. Bottom: Number of tumors and patients underlying each box. For the activity concentration, G1, two outliers at cycle $1(6.8$ and $7.5 \mathrm{MBq} / \mathrm{ml})$ are excluded. 


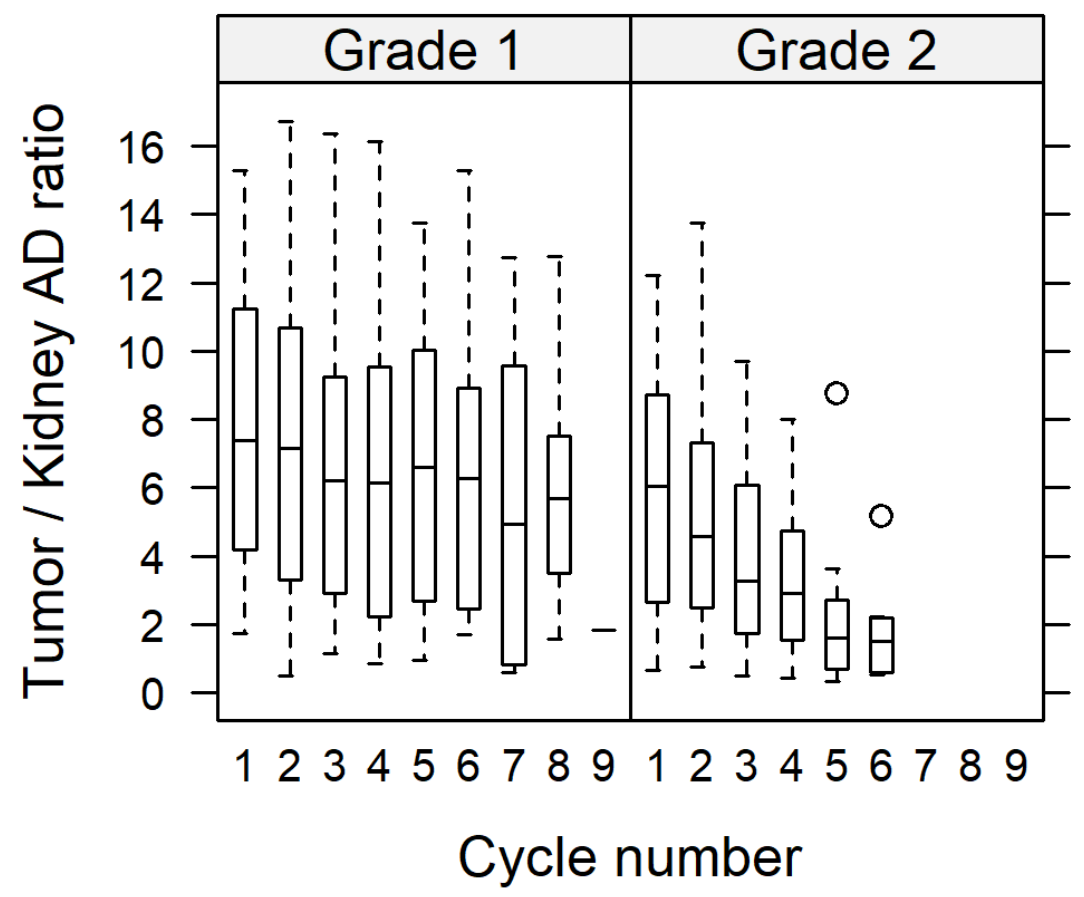

Figure 7. Ratio of the AD to tumor and kidneys (mean left and right) for G1 and G2 NETs. 


\section{Graphical Abstract}

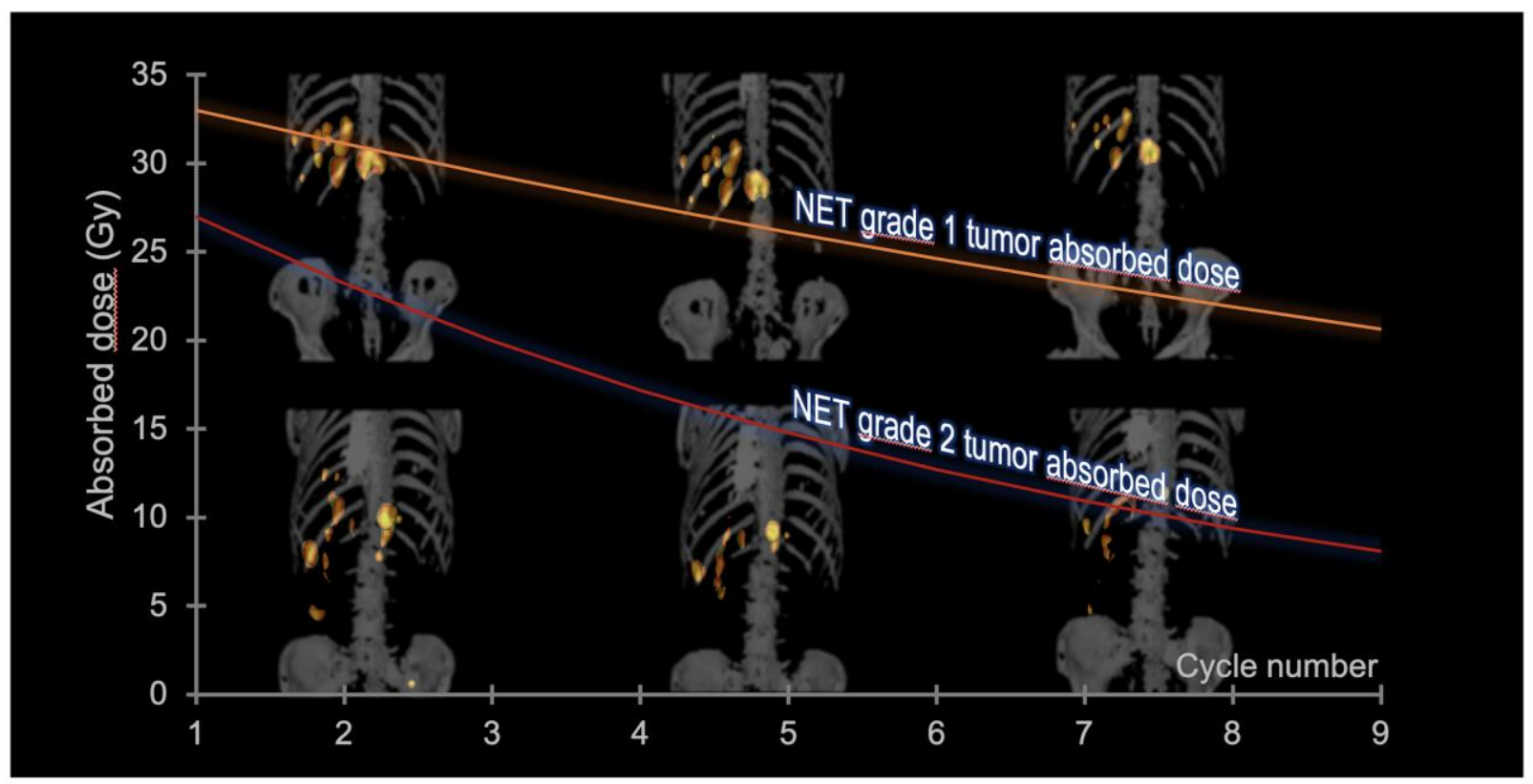


Daniel Roth, Johan Gustafsson, Carl Fredrik Warfvinge, Anna Sundlöv, Anna Åkesson, Jan Tennvall, Katarina Sjögreen Gleisner

\section{SUPPLEMENT 1}

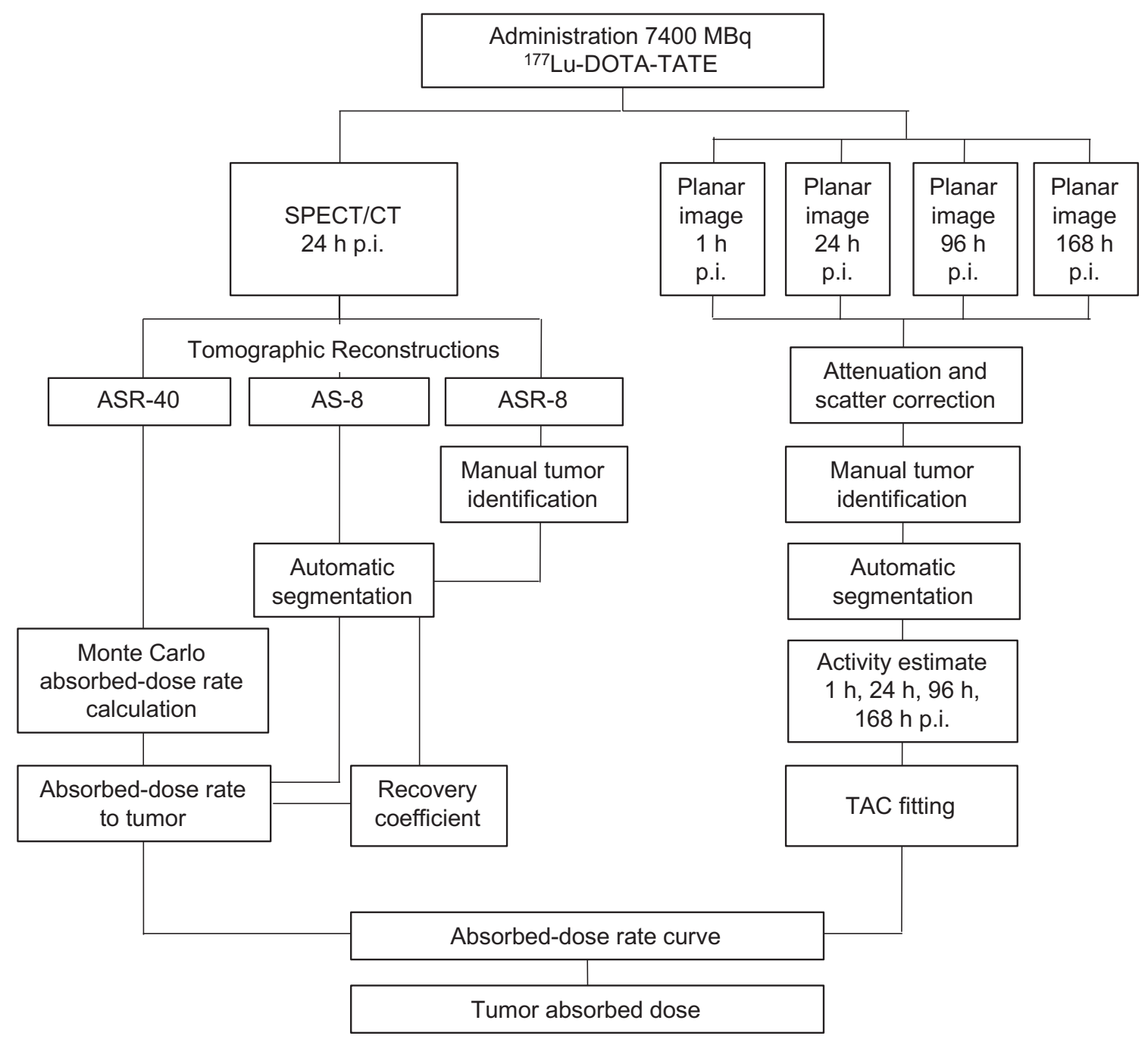

FIGURE A1. Schematic overview of the process for image processing and analysis. 
Daniel Roth, Johan Gustafsson, Carl Fredrik Warfvinge, Anna Sundlöv, Anna Åkesson, Jan Tennvall, Katarina Sjögreen Gleisner

\section{SUPPLEMENT 2}

The distribution of tumor volumes at cycle 1 are shown in Figure A2. The median volumes were $10 \mathrm{ml}$ (range $2 \mathrm{ml}$ to $480 \mathrm{ml}$ ) for $\mathrm{G} 1$ and $9 \mathrm{ml}$ (range $2 \mathrm{ml}$ to $794 \mathrm{ml}$ ) for $\mathrm{G} 2$. The fixed-effect intercepts from the LMM with volume as dependent variable (Eq. 1) were $20 \mathrm{ml}$ and $16 \mathrm{ml}$ for $\mathrm{G} 1$ and $\mathrm{G} 2$ patients, respectively, and were not significantly different $(\mathrm{p}=0.4)$.
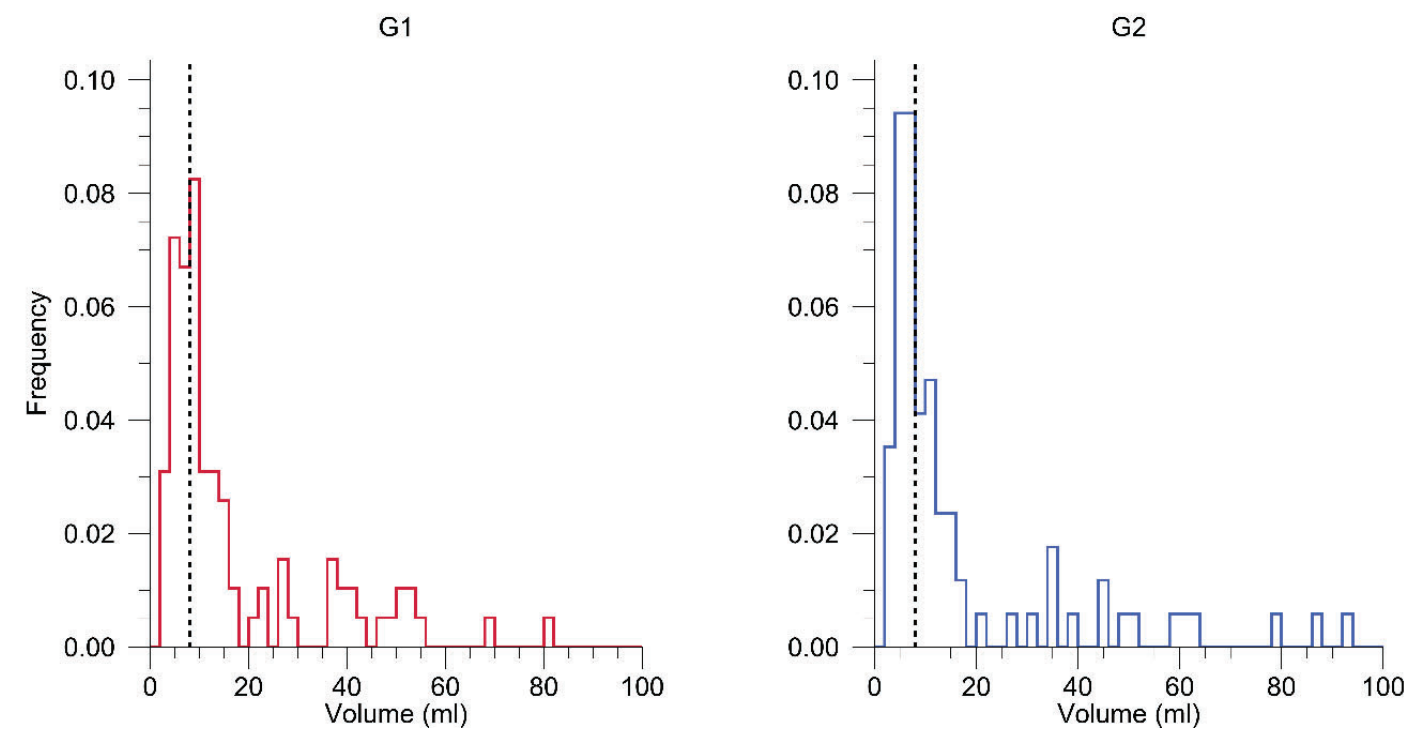

Figure A2. Frequency of G1 (left) and G2 (right) tumors at cycle 1. Dashed vertical line indicates the volume cutoff set at $8 \mathrm{ml}$. For visibility the horizontal axis has been truncated at $100 \mathrm{ml}$, omitting ten tumors with larger volumes for G1 $(n=5)$ and $\mathrm{G} 2(\mathrm{n}=5)$. 
Dosimetric quantities of neuroendocrine tumors over treatment cycles with ${ }^{177}$ Lu-DOTA-TATE

Daniel Roth, Johan Gustafsson, Carl Fredrik Warfvinge, Anna Sundlöv, Anna Akesson, Jan Tennvall, Katarina Sjögreen Gleisner

\section{SUPPLEMENT 3}

Results of the cumulative AD over the given cycles for the 65 tumors that had complete dosimetry and fulfilled the volume criterion for all cycles. The alternative methods are listed in Table 2 in the main paper. Alternatives (A, B, C) are intended as possible simplifications of the dosimetry protocol and include assumptions of (A) constant absorbed dose per cycle estimated from first cycle, (B) constant effective half-time estimated from first cycle, $(\mathrm{C})$ constant effective half-time estimated as the global means from the LMM of $103 \mathrm{~h}$ (grade 1) and $81 \mathrm{~h}$ (grade 2). Method (D) represents the use of the LMM to interpolate from existing data for cycles where the volume criterion discriminated the data for some cycles. Method (E) represents results of the complete LMM, and deviations thus represent the residuals of the LMM fit.

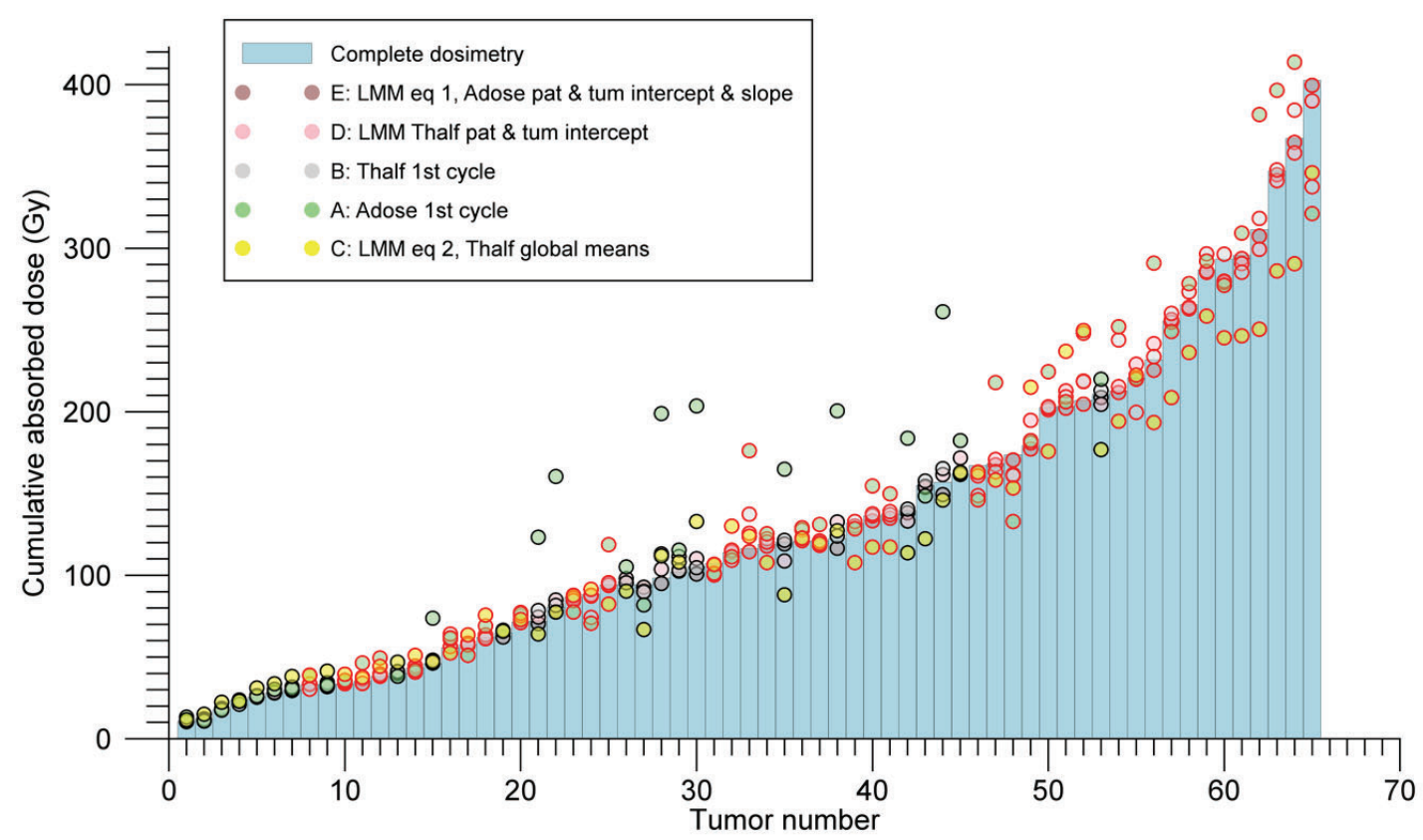

FIGURE A3. Bars show the cumulative absorbed dose over the given cycles for 65 tumors for which all the adapted criteria were fulfilled in all cycles. Symbols for G1 tumors are outlined in red, G2 in blue. Symbol fill colors indicate the calculated cumulated absorbed dose when invoking the calculation methods listed in Table 2, corresponding to A - green, B - gray, C - yellow, D - pink and $\mathrm{E}$ - brown. 
Daniel Roth, Johan Gustafsson, Carl Fredrik Warfvinge, Anna Sundlöv, Anna Åkesson, Jan Tennvall, Katarina Sjögreen Gleisner

Analysis of the relative difference between the cumulative AD calculated with complete dosimetry and different alternative methods for 65 tumors. The relative difference is calculated as: (AD for simplified protocol / AD from complete dosimetry -1$)$. The limits of agreement are defined as two standard deviations (SDs) of the relative difference. The SD is estimated as a one-way analysis of variance, calculated as a combination of intra- and inter-patient variability.

A

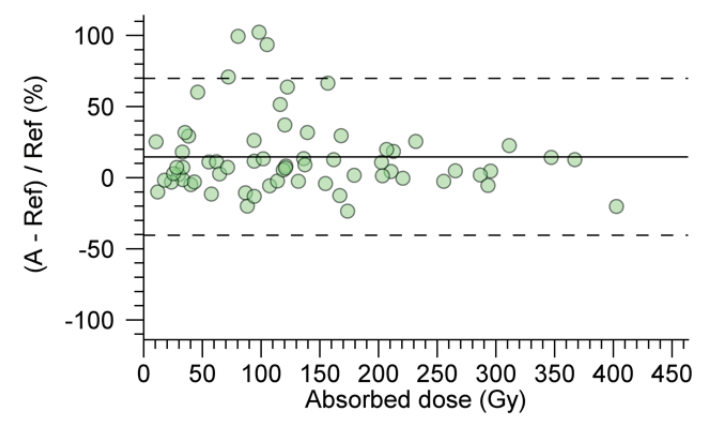

$\mathrm{C}$

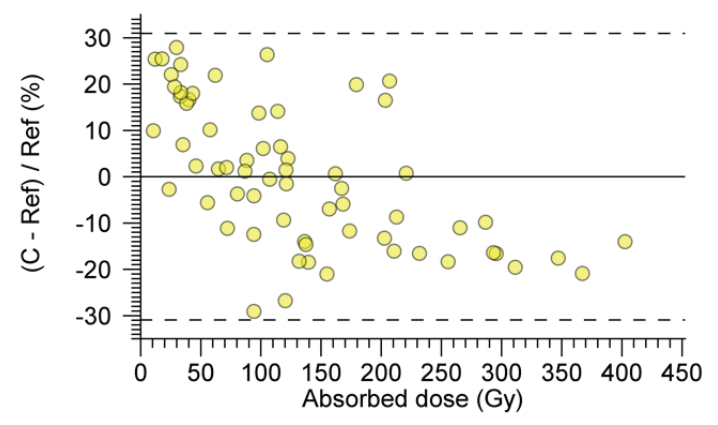

$\mathrm{E}$

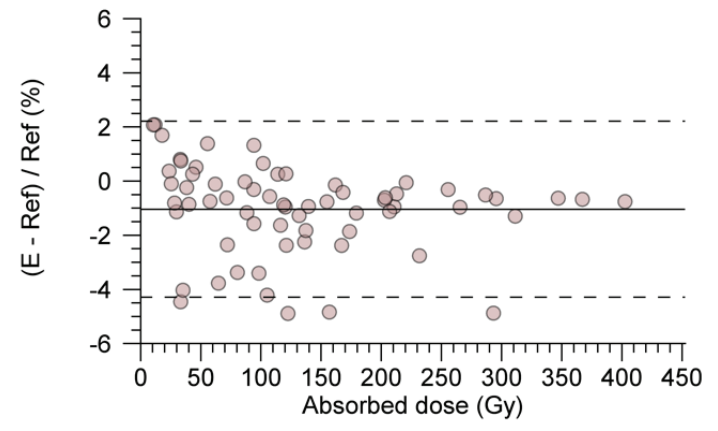

$\mathrm{B}$

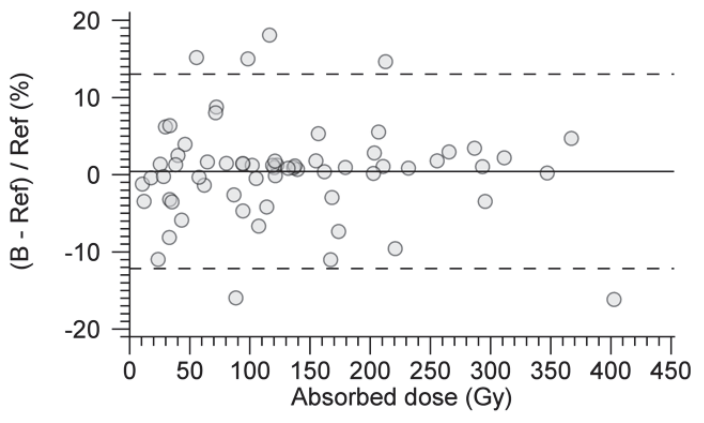

$\mathrm{D}$

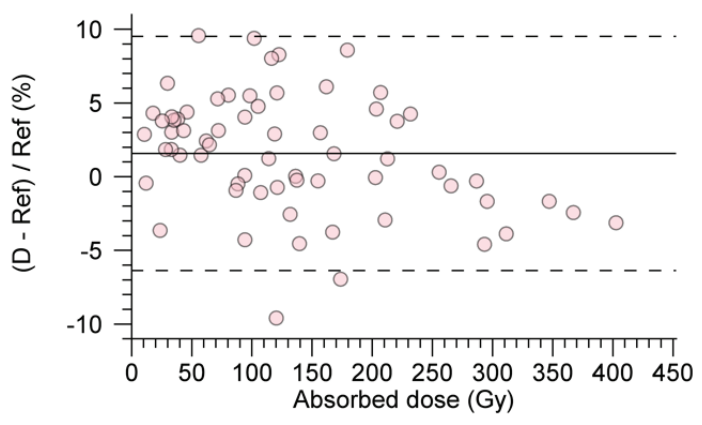

Figure A4. Bland-Altman plots of the relative difference in the cumulative AD between the alternative dosimetry methods, and the reference, i.e., the AD calculated by complete dosimetry for all given cycles. Panel letters and symbol colors follow those defined in Figure A3. Solid lines indicate mean deviation, dashed lines the limits of agreement. Note the different ranges on the vertical axes. 